\title{
Aplicação de Rede Social no Manejo da Hipertensão
}

\section{Applying social network in the management of hypertension}

\author{
Angela Beatriz Papaleo Wagner, MD; Hamilton Lima Wagner, MD; Yves Roland Talbot, MD; \\ Anousca Evelin do Carmo
}

\section{Resumo}

Os autores revisam a literatura sobre redes sociais e aplicando a metodologia de terapia de grupos trabalham com uma coorte de hipertensos, sem grupo controle, na tentativa de melhorar o controle da patologia. Os resultados obtidos neste trabalho inicial parecem promissores, indicando a necessidade de um novo estudo multicentrico para permitir uma melhor avaliação do impacto desta técnica terapêutica no controle de doenças crônicas como a hipertensão arterial sistêmica.

Palavras chaves: rede social e hipertensão.

\begin{abstract}
In this study, the authors have reviewed the literature about social networks. Applying the methodology of group therapy, they work with a cohort of hypertensive patients, but without a control group, and searching to improve the disease follow up. The results obtained in this initial study seem very interesting, showing the need of a new multi-centric study to allow a better evaluation of the impact of this therapeutic tool in the control of chronic diseases, such as the systemic arterial hypertension.
\end{abstract}

Key words: social network and hypertension

\section{Introdução}

O trabalho na atenção à saúde freqüentemente esbarra na falta de apoio da família e da sociedade para que seja encontrada uma melhor solução ao problema em foco. Principalmente quando o problema fere os padrões aceitos pelo grupamento, gerando culpas e ansiedades.

A compreensão dos valores da sociedade, sua forma de organização e recursos disponíveis são fonte básica para que a atenção à saúde se faça com conseqüência e lógica, obtendo resultados duradouros. Isto é muito mais evidente quando os problemas envolvem credos familiares ou ainda medos sociais como nas doenças mentais.

Num estudo da sociedade à qual se está inserido, é importante compreender e avaliar os recursos que a comunidade possui, organizações que podem somar na busca da solução de problemas identificados e em como acessar estes serviços. Este tipo de contato otimiza os recursos disponíveis e acelera a solução dos problemas a nível local.

Quando se enfrenta situações afetas a um indivíduo ou a uma família, deve-se identificar que tipos de apoio estão disponíveis, sejam eles familiares, profissionais, sociais ou de serviço. Qual a proximidade e disponibilidade destes recursos, e em como se pode mobilizá-los. Isto fornece à equipe de saúde uma noção melhor das dificuldades que se encontrará numa dada situação e qual o melhor caminho para buscar a solução.

Uma vez traçado um mapa dos recursos, o profissional pode desenvolver um armamentário apropriado de recursos que torne mais eficiente o seu trabalho, permitindo-lhe buscar os recursos identificados como necessários e oferecendo à comunidade uma resolutividade maior.

Os conhecimentos da organização social, seus recursos e disponibilidades permitem que a equipe obtenha resultados surpreendentes (que se mantém ao longo do tempo), e favorece uma ação coordenada e racional, apoiando-se nas fontes existentes para otimizar o esforço.

\section{Material e métodos}

A partir de uma revisão de literatura, foram explorados os impactos das redes sociais no processo saúde doença, bem como a estratégia de desenvolver redes de apoio e técnicas de terapia de grupo, como formas de promoção à saúde.

A técnica desenvolvida foi, a partir de atividades programáticas, envolver pacientes e seus familiares num trabalho de grupo terapêutico, utilizando dinâmicas de recepção, regras de convivência, elencando problemas trazidos e selecionados pela comunidade e promovendo a partir disto atividades variadas de debate e trabalho em grupo. Situações de isolamento, depressão e conflitos foram abordados de modo particularizado estimulando a criação de redes comunitárias.

Aplicou-se o trabalho na comunidade atendida pela Unidade de Saúde Santa Rita da Prefeitura Municipal de Curitiba, onde através de grupos terapêuticos se desenvolveu a perspectiva de criação de redes de apoio. $\mathrm{O}$ resultado encontrado foi avaliado através de um questionário qualitativo (quadro 1) aplicado a uma amostra de usuários que participam das atividades, cotejados com os dados de prontuário médico dos mesmos (tabela 1). 0 critério de inclusão foi participar do grupo há mais de 6 meses. 
Quadro 1 - Questionário aplicado a uma amostra atendida pelo programa de hipertensos.

1. Se você tivesse algum problema, neste momento, com quem contaria para ajudá-lo (a)?

2. A sua família costuma ajudá-lo (a)?

3. Você costuma ajudar parentes, amigos, vizinhos?

4. Quem poderia ajudá-lo (a) em caso de necessidade? (vizinhos, escola, comunidade, igreja, grupos de apoio, trabalho, família, equipe de saúde, outras instituições)?

5. Você participa de algum grupo, ou conselho, ou instituição?

6. Como você contribui para melhorar sua comunidade?

7. Participar dos grupos de trabalho auxilia no estabelecimento de novas relações?

8. Poderia citar alguma solução ou relacionamento desenvolvido através de sua participação nos grupos?

9. Voltar às atividades dos grupos é decisão sua ou sente-se obrigado a voltar? Se tivesse opção participaria dos mesmos?

Tabela 1 - Controle de pacientes pelo nível da hipertensão

\begin{tabular}{|l|l|}
\hline Grau hipertensão & Controle \\
\hline 2 & Controlado \\
\hline 1 & Controlado \\
\hline 1 & Controlado \\
\hline 1 & Controlado \\
\hline 2 & Controlado \\
\hline 1 & Controle regular \\
\hline 2 & Controlado \\
\hline 2 & Controlado \\
\hline 2 & Controlado \\
\hline 1 & Controlado \\
\hline 2 & Controle regular \\
\hline 2 & Controlado \\
\hline 2 & Controlado \\
\hline 2 & Controlado \\
\hline 1 & Controlado \\
\hline 1 & Controlado \\
\hline 1 & Controle regular \\
\hline 3 & Descontrolado \\
\hline 2 & Descontrolado \\
\hline
\end{tabular}

PA grau 1: diastólica 90-99 mmHg e sistólica 140-159 $\mathrm{mmHg}$

PA grau 2: diastólica 100-109 mmHg e sistólica 160-179 $\mathrm{mmHg}$

PA grau 3: diastólica $\geq$ $110 \mathrm{mmHg}$ e sistólica $\geq 180$

A classificação é feita sobre o pior dado.
PA controlada $=$ dentro dos valores ditos normais. PA com controle regular $=$ valores equivalentes a HAS grau 1.

PA descontrolada $=$ valores acima dos valores atribuídos acima.

Em 19 pacientes que participaram da entrevista, 14 estavam controlados (74\%), 3 estavam com um controle regular (16\%) e apenas $2(10 \%)$ não se encontravam controlados.

\section{Revisão de Literatura}

A compreensão das maneiras de interagir que as pessoas desenvolvem, características que vem dos modelos materno (sobrevivência), paterno (regra) e casal (convivência), abre uma ampla perspectiva de trabalho com grupos e famílias. O modelo sistêmico integrativo proposto por Grassano explora as características individuais na composição de uma relação. Quando se analisa o funcionamento da família proposto por Minuchin e integrando os conceitos, fica fácil identificar como os comportamentos da família de origem se repetem e interferem nas atitudes da família e sua ação dentro da comunidade.

Sluski, em seu livro sobre redes sociais, explora os efeitos das relações interpessoais e em como isto interfere no processo saúde-doença. As pessoas foram analisadas em função do tamanho e qualidade da rede, tendo sido demonstrado que redes equilibradas e eficientes estão ligadas a melhor qualidade de vida e menor morbi-mortalidade.

Barreto, em seu trabalho de psiquiatria comunitária, desenvolveu uma metodologia de trabalho em grupos, no qual explorando as experiências pessoais dos participantes dos grupos busca-se um encaminhamento coletivo, desenvolvendo parcerias e cumplicidade entre a equipe de saúde e a população atendida. Explorando os aspectos positivos, busca as forças intrínsecas da população, permitindo que as redes trabalhadas por Sluski ocorram de uma maneira natural, facilitando o controle de situações de estresse.

Papp explora em como as agendas pessoais, características de gênero e modos de enfrentar as 
diversas situações podem influir no processo, o que permite que tenhamos uma estrutura de trabalho considerando as características do grupo. Isto conecta as pessoas na realidade diária e nas suas relações familiares e comunitárias, dando uma ferramenta interessante para estimular o debate dentro da comunidade.

Ausloss aborda a competência e conhecimento que as pessoas e famílias têm de suas histórias, e em como isto pode ser relacionado com a construção de alternativas viáveis - o que enfoca a necessidade de valorizar suas iniciativas e soluções na busca de um melhor modo de viver, ao mesmo tempo em que ouvindo a voz dos outros resolvendo seus próprios problemas, encontram caminhos próprios e tem sua auto-estima melhorada.

Freire discute a necessidade de reconhecer a realidade das pessoas e como construir o conhecimento a partir de seus pontos de partida, respeitando-lhes o caminho e oferecendo opções conectadas com a realidade. Só um aprendizado conectado à realidade $\mathrm{e}$ concreto pode ser incorporado, isto é chave para um trabalho comunitário. Os trabalhos de Ausloss e Freire são complementares na proposta de ações com a comunidade, e a técnica de Barreto cria um espaço que explora os potenciais da mesma - esta combinação, explorada nesse trabalho, baseia-se neste tripé.

Carter explora as diversas fases da vida e em como estas fases interferem na qualidade de vida - que tarefas precisam ser cumpridas para uma adequada resolução dos diversos momentos e Wagner coloca a necessidade de facilitar a compreensão destas tarefas e em como a equipe de saúde pode atuar para que a comunidade perceba melhor os seus pontos de estrangulamento. Explorando o desenvolvimento da comunidade e crescendo passo a passo, como proposto por Freire, pode-se centrar o trabalho no momento de cada grupo em questão.

McDaniel cita diversos níveis de intervenção a nível familiar, onde a aplicação de seus conceitos e dos instrumentos de trabalho auxilia na comunicação com os grupos, permitindo um melhor entrosamento e compreensão tanto da comunidade como da equipe de trabalho.

Wagner explora os princípios de trabalho em atenção primária e em como os conceitos sistêmicos propostos por Christie-Seely podem ser aplicados nessa área de atuação. Aqui são consideradas as ações de prevenção associadas à atenção e se percebe a doença dentro de um contexto particular de vida experienciado pelo paciente e seus relativos.

Talbot explora os instrumentos de estudo e facilitação da família, enfocando técnicas de abordagem sistêmica. Propõe maneiras de se associar à família e auxiliar no encaminhamento de seus problemas, além de trabalhar técnicas de exercício em grupo que enormemente facilitam a incorporação de novos pontos de vista.

Rogers, em seu excelente "Tornar-se Pessoa", enfatiza a necessidade de compreender os caminhos para o crescimento pessoal e associa as dinâmicas que movem as famílias de uma dimensão do trabalho de resgate da dignidade e valorização do caminho seguido pelos indivíduos que os trouxe até aqui. Explora os limites que partem das crenças pessoais e grades auto criadas, mesmo assim reais e de difícil mobilização.

\section{Discussão}

Inúmeros trabalhos - saúde e mortalidade (2), cardiovascular (5 e 21), resultado da gestação (9), educação de filhos (7 e 28), imunidade (15), câncer (24 e 27), saúde mental (30 e 31), odontologia (13) -, publicados na literatura, demonstram a melhora da qualidade de vida, bem como a diminuição de índices de morbi-mortalidade, com o desenvolvimento de redes sociais - uma simples consulta ao "Institute for Scientific Information" mostrou mais de 5000 artigos sobre o tema nos últimos 7 anos! A atenção primária à saúde, que tem o viés de incorporar prevenção e atenção à saúde, não poderia desconsiderar todo este referencial.

Quando se usa o referencial sistêmico e se compreende a saúde com este novo olhar, se percebe que, se em um nível há problemas, o deslocamento da ação para o nível seguinte oferece boas possibilidades de solucionar esses conflitos. Considerando que o lócus de ação da atenção primária vai da pessoa, passa pela família e estende-se à comunidade, fica óbvio que trabalhar com a comunidade pode ser uma grande opção para melhorar os resultados.

Dentro dessa perspectiva iniciou-se uma série de atividades com o intuito de perceber as necessidades da comunidade, seus modos peculiares de interagir e em como os conceitos estudados se manifestavam dentro da comunidade. É preciso compreender as diferenças entre a população estudada pela maioria dos autores, de origem norte-americana ou européia, e a realidade da comunidade do Tatuquara, bairro da periferia de Curitiba onde se insere a unidade de saúde Santa Rita.

A comunidade assentada na área de influência da unidade é oriunda de outras regiões de Curitiba ou do interior do estado do Paraná. As famílias foram relocadas através de um loteamento da COHAB (Companhia de Habitação) de Curitiba. Fazem parte da população de classe média baixa, com nível médio de escolaridade (ensino fundamental incompleto). A população é formada, em grande parte por trabalhadores domésticos ou da construção civil, além de muitos comerciários. A comunidade é atendida por três escolas municipais, que oferecem ensino fundamental, inclusive com turmas para adultos. A municipalidade mantêm na área cursos profissionalizantes e ginásio público de esportes, há um distrito policial, serviço de acompanhamento de jovens em contra-turno da escola, além de várias creches para atendimento a comunidade. A população estimada da área é de 20000 habitantes. A área é relativamente nova, com menos de 10 anos de assentamento, o que também dá uma peculiaridade da falta de referências históricas e lealdade dentro da comunidade. 
Os índices de violência, com assaltos, assassinatos, tráfico de drogas e alcoolismo são elevados, acima dos índices médios da cidade de Curitiba, o que gera muita ansiedade na população. A área fica longe das demais áreas urbanizadas de Curitiba, fazendo com que o acesso a serviços de saúde seja muito difícil; a exceção é a unidade de saúde Santa Rita, que trabalha com uma sobrecarga de serviço, demora no atendimento e estresse pela concentração de pessoas em uma área restrita para obtenção de serviço.

Dentro desta realidade, as atividades programadas são uma necessidade para facilitar o atendimento de situações elencadas como prioritárias; aproveitando a estrutura dada pela Secretaria Municipal de Saúde de Curitiba, iniciou-se uma rediscussão destas atividades, incorporando famílias e comunidade em cada uma delas. Usando a reflexão oriunda do pensamento sistêmico, se provocou na comunidade a curiosidade sobre seu próprio fazer e modo de se relacionar. Buscando identificar dificuldades e facilitando a colocação de conceitos básicos, rediscutem-se os papéis a partir do modelo sistêmico integrativo. Usando a base teórica para provocar discussões, abre-se um grande leque de interação em que as pessoas param e refletem sobre suas vidas, seus relacionamentos e em como isto influencia a qualidade de suas vidas.

Sluski identifica quatro grandes áreas em que as relações se estruturam: família, amizades, trabalho e instituições. Cada uma destas áreas deve ser considerada no reconhecimento da existência de rede social, avaliando proximidade e disponibilidade, fatores que identificam o grau de proteção que a rede provê a uma dada pessoa. As redes são interdependentes e muitas vezes, apesar de possuírem um grande número de pessoas, são de difícil mobilização, o que as torna inoperantes. Este foco também é explorado nas atividades, permitindo que a comunidade se aperceba e vá estabelecendo suas próprias redes espontâneas. O trabalho desenvolvido considera as necessidades afetivas e de suporte, o que estreita os laços de união da comunidade, favorecendo a continuidade do trabalho.

A metodologia aplicada às reuniões, fruto do estudo do material desenvolvido na Universidade Federal do Ceará pelo Professor Adalberto Barreto, é simples, mas muito eficiente. Uma série de dinâmicas de acolhimento é feita, permitindo que a população sinta-se integrada e bem vinda. Regras de convivência simples são acordadas, como respeitar a fala do outro, manter sigilo das falas apresentadas - o que permite que as pessoas possam se abrir e colocar suas experiências. Também é acordado de que se fale apenas de experiências pessoais, de modo que se evite o aconselhamento - que pode ter efeitos nocivos no processo - permitindo assim que se aprenda pelo modo de vida do outro e se aproveite apenas o que tem lógica em nosso próprio referencial. A equipe de saúde trabalha como mediadora, evitando ser a dona do saber, mas uma catalisadora das idéias e temas propostos pela comunidade. Cabe à equipe redimensionar problemas, mostrando as competências dos atores envolvidos, reafirmando seus caminhos enquanto propõe questões a serem desenvolvidas pelo grupo. A atividade busca sempre deixar questões a serem trabalhadas pelo grupo, num contínuo de reflexão e crescimento.

No novo paradigma sistêmico para prevenção e tratamento de doenças e incapacidades se faz importante incorporar modos de promover o suporte social e o desenvolvimento da família. Isto fortalece a intervenção da equipe de saúde, e também há um suficiente corpo de evidências que justifica isto, apesar dessas evidências serem praticamente ignoradas pelos profissionais que ainda se fixam no atendimento individual de pacientes.

Momentos chaves do ciclo de vida das famílias são provocados como tema de reflexão, auxiliando os participantes a identificarem situações críticas, em que a saúde pode ser perturbada, e em como isto pode ser mais bem enfrentado através do cumprimento das tarefas apropriadas em cada fase. Interessantes discussões ocorrem quando temas trazidos se situam nos momentos críticos do ciclo de vida familiar; experiências variadas e soluções criativas surgem espontaneamente - totalmente identificadas com a realidade da comunidade e permitindo que a comunidade se identifique como atriz do seu processo. Ocorre o resgate da auto-estima e se fomentam referências dentro da própria comunidade. Isto muda o referencial, onde a equipe de saúde é a provedora da saúde, para um papel de co-responsabilidade em que a população assume o fundamento de que as ações pessoais estão diretamente ligadas ao seu processo de vida e sua saúde. Os participantes dos grupos são unânimes em afirmar que sua participação nos mesmos é algo gratificante e que se sentem convidados a comparecer sempre que possível, pois cada encontro é razão para encontrar amigos. Trinta e sete por cento deles referem-se à equipe de saúde para enfrentar seus problemas, e apenas 1 (5\%) não se vê envolvido em tarefas de auxílio a questões da comunidade.

A equipe recebe a demanda de fornecer respostas claras a dúvidas levantadas pela comunidade, o que é muito diferente de falar sobre algo de interesse da equipe de saúde. Cada encontro torna-se rico e permite à comunidade trabalhar temas que lhe são peculiares e estão no momento chamando a atenção. Torna os encontros atrativos, fazendo com que a frequiência aos mesmos seja cada vez maior. Fatos interessantes como a freqüência masculina em programas de planejamento familiar e pré-natal, avós e outros cuidadores nos programas voltados a crianças e lactentes, professores e outros educadores participando das atividades junto com pais de alunos para discussão de educação e relacionamento conjugal, são alguns dos indicadores da receptividade da comunidade para a proposta de trabalho.

Toda esta atividade vem produzindo um volume de relacionamentos de rede, identificando situações semelhantes em que é possível espelhar-se, e mais do que tudo criando vínculos comunitários mais saudáveis e funcionais. 


\section{Conclusão}

Trabalhar com o conceito de rede social, utilizando grupos terapêuticos como forma de concretização da construção de redes é uma forma de agilizar o princípio da educação de adultos dentro da atenção primária. Além de extremamente gratificante para a equipe, que passa a ser identificada não mais como mera provedora de atenção à saúde, mas como parceira da comunidade na busca de uma melhor qualidade de vida.

Independentemente de quaisquer resultados clínicos o simples fato de fazer as pessoas reconhecerem suas competências e sentirem-se valorizadas no seu fazer já é um resultado absolutamente satisfatório para o trabalho desenvolvido. A parceria estabelecida também diminui a pressão sobre a equipe de saúde no que tange à demanda, permitindo um trabalho mais harmônico e de qualidade.

Mas o resultado clínico também é muito significativo, o número de descompensações em doenças crônicas reduziu-se. Dados da Organização Mundial da Saúde referem que 50\% dos hipertensos não são controlados, e nos $50 \%$ restantes a metade tem um controle insatisfatório. A aderência aos tratamentos propostos aumentou e a comunidade sente-se confiante para conversar sobre dificuldades que podem comprometer os seus cuidados, o que fez com que na população estudada obtivéssemos um índice de $74 \%$ de bons resultados.

Os autores analisam o trabalho desenvolvido como uma importante alternativa na atenção à saúde, e a busca do desenvolvimento de redes sociais como uma meta para as equipes de atenção primária. Isso permite uma melhor utilização dos recursos próprios da comunidade, uma maior satisfação da comunidade e da equipe de saúde com o trabalho desenvolvido, sem que signifique mais carga de trabalho ou custo adicional.

A aplicação do pensamento sistêmico e do conceito de redes sociais se torna fundamental para uma atenção primária mais eficiente e resolutiva. Os dados obtidos neste trabalho sugerem a necessidade de um estudo mais abrangente e multicêntrico para que se estabeleça uma melhor análise.

\section{Bibliografia}

1. ACHAT, H.; KAWASHI, I.; LEVINE, S.; BERKEY, C.; COAKLEY, E.; COLDITZ, G. Social network, stress and health-related quality of life. Quality of Life Research. 1998; 7(8): Pg. 735-50.

2. ANTONUCCI, T. C.; AJROUCH, K. J.; JANEVIC, M. Socioeconomic status, social support, age, and health. In: Socioeconomic status and health in industrial nations. 1999. Pg. 390-2.

3. AUSLOSS, G. A competência das famílias. Lisboa: Climepsi. 1996.

4. BERKMAN, L. F. Role of social-relations in health promotion. Psychosomatic Medicine. 1995; 57(3): Pg. 245-54.
5. BROADWELL, S. D.; LIGHT, K. C. Family support and cardiovascular responses in married couples during conflict and other interactions. International Journal of Behavioral Medicine. 1999; 6(1): Pg. 40-63.

6. CARTER, B; McGOLDRICK, M. et al. As mudanças no ciclo de vida familiar. 2.ed. Porto Alegre: Artes Médicas. 1995. p.7-29.

7. CHEEVER, K. H.; HARDIN, S. B. Effects of traumatic events, social support, and self-efficacy on adolescents' self-health assessments. Western Journal of Nursing Research. 1999; 21(5): Pg. 673-84.

8. CHRISTIE-SEELY, J. et all. Working with families in primary care. Oxford. 1984.

9. COLLINS N. L.; DUNKELSCHETTER, C.; LOBEL, M.; SCRIMSHAW, S. C. M. social support in pregnancy - psychosocial correlates of birth outcomes and postpartum depression. Journal of Personality and Social Psychology. 1993; 65(6): Pg. 1243-58.

10. DUE, P.; HOLSTEIN, B.; LUND, R.; MODVING, J.; AVLUNG, K. Social relations: network, support and relational strain. Social Science \& Medicine. 1999; 48(5): Pg. 661-73.

11. FREIRE, P. Pedagogia da esperança. 10. ed. Rio de Janeiro: Paz e Terra. 2003.

12. GRASSANO, S. et all. Abordagem sistêmico integrativa. CD Rom - Chaim. Curitiba. 1996.

13. HANSON, B. S.; LIEDBERG, B.; OWALl, B. Social network, social support and dental status in elderly Swedish men. Community Dentistry and Oral Epidemiology. 1994; 22(5):331-7.

14. HERRMANN, M. M.; VAN CLEVE, L.; LEVISEN, L. Parenting competence, social support, and selfesteem in teen mothers case managed by public health nurses. Public Health Nursing. 1998; 15(6):4329

15. KANG, D. H.; COE, C. L. KARASZEWSKI, J. McCARTHY, D. O. Relationship of social support to stress responses and immune function in healthy and asthmatic adolescents. Research in Nursing \& Health. 1998; 21(2):117-28

16. LEKANDER, M.; FURST, C. J.; ROSTEIN, S.; BLOMGREN, H.; FREDRIKSON, M. Social support and immune status during and after chemotherapy for breast cancer. Acta Oncologica. 1996; 35(1): 31-7.

17. LEPORE, S. J.; ALLEN, K. J. JANEVIC, M. Social support lowers cardiovascular reactivity to an acute stressor. Psychosomatic Medicine. 1993; 55(6): 518-24.

18. McDANIEL, S. H. et ali. Terapia familiar médica. Porto Alegre:Artes Médicas, 1994.

19. McEWEN, B. S.; STELLAR, E. Stress and the individual mechanisms leading to disease. Archives of Internal Medicine. 1993; 153(18): 2093-101.

20. MINUCHIN, S. Famílias, funcionamento \& tratamento. Porto Alegre: Artes Médicas. 1990. p. 52-69.

21. ORTHGOMER, K.; ROSENGREN, A.; WILHELMSEN, L. Lack of social support and incidence of coronary heart-disease in middle-aged Swedish men. Psychosomatic Medicine. 1993; 55(1): 37-43

22. PAPP, P et al. Casais em perigo. Porto Alegre: Artmed. 2002.

23. RAEL E. G. S.; STANSFELD, S. A.; SHIPLEY, M.; HEAD, J.; FEENEY, A.; MARMOT, M. Sickness absence in the Whitehall-Ii study, London - the role of the social support and material problems. Journal of 
Epidemiology and Community Health. 1995; 49(5): 474-81

24. REYNOLDS, P.; BOYD, P. T.; BLACKLOW, R. S. JACKSON, J. S.; GREENBERG, R. S.; AUSTIN, D. F. et ali. The relationship between social ties and survival among black and white breast-cancer patients. Cancer Epidemiology Biomarkers \& Prevention. 1994; 3(3): 253-9

25. ROGERS, C Tornar-se pessoa. 7. ed. Lisboa: Moraes Editores. 1985.

26. RYFF, C. D.; SINGER, B. Interpersonal flourishing: a positive health agenda for the new millennium. Personality and Social Psychology Review. 2000; 4(1): $30-44$.

27. SEOW A.; HUANG, J.; STRAUGHAN P. T. Effects of social support, regular physician and health-related attitude on cervical cancer screening in an Asian population. Cancer Causes \& Control. 2000; 11(3): 223-30.

28. TARKKA, M. T.; PAUNONEN, M.; LAIPPALA, P. Social support provided by public health nurses and the coping of first-time mothers with child care. Public Health Nursing. 1999; 16(2): 114-9.

29. THEORELL, T.; BLOMKVIST, V.; JONSSON, H.; SCHULMAN, S.; BERNTORP, E.; STIGENDAL, L. Social support and the development of immune function in human-immunodeficiency-virus infection. Psychosomatic Medicine. 1995; 57(1): 32-6.

30. UCHINO, B. N.; CACIOPPO, J. T., KIECOLTGLAESER, J. $\mathrm{K}$. The relationship between social support and physiological processes: a review with emphasis on underlying mechanisms and implications for health. Psychological Bulletin. 1996; 119(3): 32-6.

31. UCHINO, B. N.; UNO, D.; HOLT-LUNSTAD, J. Social support, physiological processes, and health. Current Directions in Psychological Science. 1999; 8(5): 145-8.

32. VANDERVOORT, D. Quality of social support in mental and physical health. Current Psychology. 1999; 18(2): 205-22

33. WAGNER, H. L; WAGNER, A. B. P. et al. Bases conceituais de trabalho em saúde da família. Curitiba. Revista Médica do Paraná. 1999. v. 57 n.1/2: 16-22.

Angela Beatriz Papaleo Wagner é médica de família e comunidade da Prefeitura Municipal de Curitiba e docente do Instituto inFamilia de Curitiba.

Endereço para correspondência: Angela Beatriz Papaleo Wagner

Rua Jaime Veiga, 170 - CEP 80310-570

Curitiba, PR

E-mail: hamiltonw@terra.com.br 